\title{
Influence of domain wall pinning on the dynamic behavior of magnetic vortex structures: Time-resolved scanning x-ray transmission microscopy in NiFe thin film structures
}

\author{
A. Vansteenkiste, ${ }^{1}$ J. De Baerdemaeker, ${ }^{1}$ K. W. Chou, ${ }^{2}$ H. Stoll, ${ }^{2}$ M. Curcic, ${ }^{2}$ T. Tyliszczak, ${ }^{3}$ G. Woltersdorf, ${ }^{4}$ C. H. Back ${ }^{4}$ \\ G. Schütz, ${ }^{2}$ and B. Van Waeyenberge ${ }^{1}$ \\ ${ }^{1}$ Department of Subatomic and Radiation Physics, Ghent University, Proeftuinstraat 86, 9000 Gent, Belgium \\ ${ }^{2}$ Max Planck Institute for Metals Research, Heisenbergstrasse 3, 70596 Stuttgart, Germany \\ ${ }^{3}$ Advanced Light Source, LBNL, Berkeley, California 94720, USA \\ ${ }^{4}$ Institut für Experimentelle und Angewandte Physik, Universität Regensburg, 93040 Regensburg, Germany \\ (Received 31 October 2007; revised manuscript received 21 January 2008; published 22 April 2008)
}

\begin{abstract}
Artificial domain wall pinning sites were created in micron-sized thin-film $\mathrm{Ni}_{80} \mathrm{Fe}_{20}$ structures, and their influence on the vortex dynamics was investigated by using time-resolved scanning transmission $\mathrm{x}$-ray microscopy. The domain wall pinning sites were introduced by means of focused ion beam etching in the form of antidots. The vortex gyration frequency increased in square-shaped structures but not in similarly modified disk-shaped structures, where no domain walls are present. This demonstrates that the domain wall pinning is causing the increased frequency. The effect is explained by the confinement of the domain wall motion to the portion of the structure that is circumscribed by the antidots and is in agreement with micromagnetic simulations.
\end{abstract}

DOI: 10.1103/PhysRevB.77.144420

PACS number(s): 75.75.+a, 76.50.+g, 07.85.Tt

\section{INTRODUCTION}

Thin-film magnetic structures form the building blocks for many spin valve systems, such as read heads and magnetic random access memories. Nevertheless, a better understanding of the fundamental physical properties is still needed in order to improve their functionality. Specifically, the dynamic behavior in submicron sized structures is a major topic in magnetism research.

One of the configurations that appear in soft magnetic micron-sized structures, for an adequate lateral size and thickness, is a closed flux vortex configuration. The in-plane magnetization in such a square-shaped structure forms four triangular domains separated by $90^{\circ}$ Néel walls. This is also called a Landau configuration. In a disk-shaped structure, on the other hand, the magnetization curls around the center in a continuous way, in the absence of domain walls. In both cases, the magnetization turns perpendicular to the plane at the center of the structure, thus forming the vortex core and avoiding a singularity in the magnetization. ${ }^{1}$

Magnetic vortex structures are also characterized by several excitation modes. The lowest frequency mode of a vortex configuration is the gyrotropic mode, which can be excited by using an in-plane magnetic field pulse. ${ }^{2}$ Such an excitation results in a displacement of the vortex core and the consecutive gyration back to its equilibrium state. This dynamic behavior has been explained as the movement of the vortex core in its self-induced magnetostatic restoring force. ${ }^{3}$ The eigenfrequency of the gyration mode typically lies in the subgigahertz range and is dependent on the size of the structure. For disk-shaped structures, it has been theoretically and experimentally found that for given material parameters, the gyration frequency approximately scales with the geometrical aspect ratio, with only a slight dependence on the thickness and lateral size separately. ${ }^{4-6}$

On the other hand, pinning of the magnetization may lead to a deviation from this ideal resonance behavior. Compton and Crowell ${ }^{7}$ found that in films with relatively large grains, the vortex core can be trapped in a pinning potential. The pinning sites were found to have a very local range in the order of tens of nanometers and had a pronounced influence on the vortex gyration frequency at small excitation amplitudes. At larger excitation amplitudes, the vortex core was found to be detrapped and gyrated uninfluenced by the pinning site, with the eigenfrequency that is determined by the dimensions of the structure.

It is well known that magnetic defects may pin not only a vortex core but also domain walls. To gain insight on the effect of domain wall pinning on the vortex gyration mode, we have intentionally created pinning sites in the form of antidots ("holes") in Permalloy $\left(\mathrm{Ni}_{80} \mathrm{Fe}_{20}\right)$ thin-film structures by means of focused ion bean (FIB) etching. Single antidots have already previously been created near the center of vortex structures. These trap the vortex core and lead to a total suppression of the gyration movement. ${ }^{8}$ In this work, the antidots were introduced further away from the structure center to avoid complete trapping and only pin the domain walls (if present). In this way, naturally occurring domain wall pinning sites are mimicked in a controlled manner. The resonance frequencies of both modified and unmodified square- and disk-shaped structures were experimentally determined by means of time-resolved scanning transmission $\mathrm{x}$-ray microscopy (STXM) and were compared to micromagnetic simulations.

\section{EXPERIMENT}

Several Permalloy structures with a lateral size of 1.5 or $1 \mu \mathrm{m}$ and a thickness of 30,40 , or $50 \mathrm{~nm}$ were investigated. The square and disk shapes were patterned in an evaporated Permalloy film by using e-beam lithography and a lift-off process. A $10-\mu \mathrm{m}$-wide and $150-\mathrm{nm}$-thick $\mathrm{Cu}$ stripline underneath the structures was used for the magnetic excitation. The stripline and magnetic structures were deposited onto a 


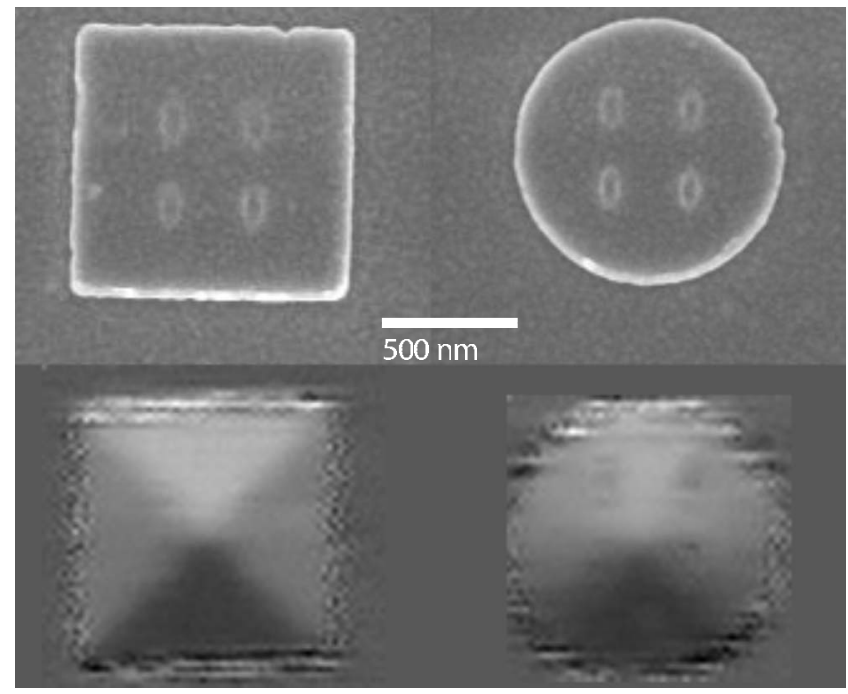

FIG. 1. Top: Scanning electron microscope images of squareand disk-shaped structures, with four antidots created by focused ion beam etching. Bottom: Corresponding STXM images showing the $x$ component of the magnetization. The domain walls of the square structure cross the antidots, while no domain walls are present in the disk.

prefabricated $100 \mathrm{~nm}$ thick $\mathrm{Si}_{3} \mathrm{~N}_{4}$ membrane. The complete sample was capped in situ with a $2 \mathrm{~nm} \mathrm{Al}$ protective layer.

Four antidots were created afterward in a number of 50nm-thick structures by means of FIB etching. An FEI Nova 600 Nanolab dual-beam FIB with a Ga ion beam at $30 \mathrm{keV}$ and $10 \mathrm{pA}$ was used. The antidots were introduced by milling away about $20 \times 40 \mathrm{~nm}^{2}$ of the Permalloy at four positions on the diagonals of the square elements. In this way, the antidots are located on the domain walls of the Landau configuration. In order to have reference measurements, identical modifications were also performed on disks (which do not have domain walls) and an additional number of structures was left unmodified. Two of the modified $1 \mu \mathrm{m}$ large Permalloy structures are shown in Fig. 1.

The magnetic vortex structures were imaged with the scanning transmission $\mathrm{x}$-ray microscope at the Advanced Light Source (ALS) (beamline 11.0.2). ${ }^{9}$ The $\mathrm{x}$-ray magnetic circular dichroism (XMCD) effect, i.e., the element specific dependence of the absorption coefficient of circularly polarized light on the direction of the magnetization in a ferromagnetic sample, ${ }^{10}$ was used as contrast mechanism for the imaging of the structures. The XMCD effect is only sensitive to the projection of the magnetization along the photon propagation direction; therefore, the sample was tilted over $60^{\circ}$ with respect to the incoming photon beam in order to observe the in-plane magnetization. The images were recorded at the $L_{3}$ absorption edge of $\mathrm{Ni}(852.7 \mathrm{eV})$. The monoenergetic photon beam is focused with a Fresnel zone plate to a spot on the sample and the transmitted intensity is detected with a point detector. A full image can be constructed by scanning the sample in the horizontal and vertical directions with a high accuracy stage. The lateral resolution is determined by the zone plate and is about $30 \mathrm{~nm}$.

Time-resolved measurements were performed in order to investigate the dynamic behavior in magnetic vortex struc- (a)

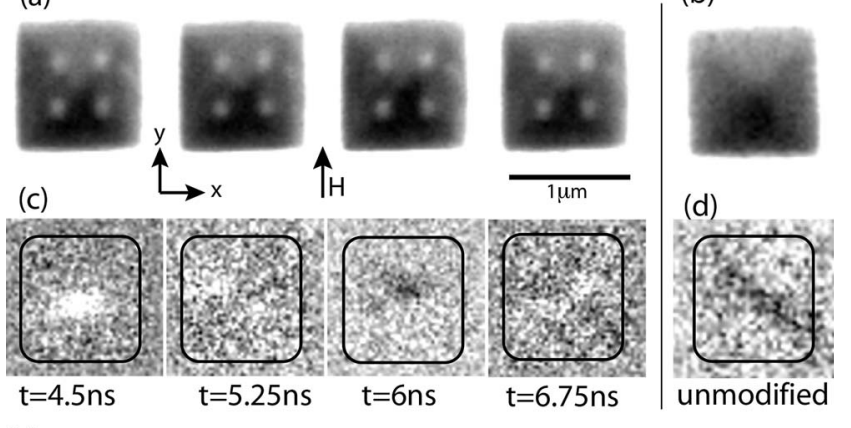

(e)

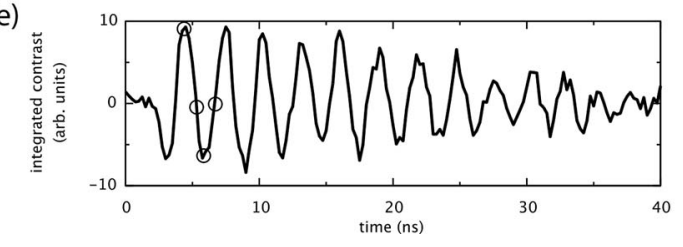

FIG. 2. (a) Sequence of STXM images for a $1 \mu \mathrm{m} \times 1 \mu \mathrm{m}$ $\times 50 \mathrm{~nm}$ modified square during one period of the oscillation. The intensity is proportional to the $x$ component of the magnetization, revealing the Landau configuration and the small gyrotropic motion of the vortex structure (gyration diameter of $\approx 100-150 \mathrm{~nm}$ ). (c) Corresponding differential image sequence that is obtained by dividing images from sequence (a) by their corresponding reference image of the configuration in the ground state. The total contrast in this sequence oscillates with the resonance frequency, as shown in (e). The four STXM images in (a) and (c) correspond to the four markers in (e). The magnetic pulse $H$ starts at $t=2 \mathrm{~ns}$. The data before the pulse correspond to the reference images of the relaxed configuration. For comparison, (b) and (d) are images of an unmodified structure corresponding to the phase of the rightmost images in (a) and (c). (d) shows that the domain wall motion can now span a larger area of the structure.

tures. The natural time structure in the storage ring of the synchrotron delivers photon flashes every 2 ns in the socalled multibunch mode. This allows the experiment to follow a typical pump-and-probe scheme, with the incoming photon flashes as probe and the externally applied in-plane magnetic field pulses as pump. While scanning each pixel, the magnetic structures were repeatedly excited every $82 \mathrm{~ns}$ by sending an electric current in the stripline underneath the structures. As each pixel is exposed for about one second, the pump procedure is repeated many times per pixel. The current pulses induce magnetic field pulses with an amplitude of about $10 \mathrm{mT}$ and a full width at half maximum of about $1 \mathrm{~ns}$ (500 ps of rise and falling time). The excitation was synchronized with the x-ray flashes of the synchrotron, which probe the magnetization at different times $t$ after the pump. A fast avalanche photodiode, which is capable of counting single photons, was used for the detection of the transmitted intensity. An appropriate acquisition scheme ${ }^{11}$ was introduced into the STXM, which is simultaneously acquiring the temporal response of the magnetization in separate channels, each of which corresponds to a different time after the pump pulse. Currently, 12 such counting channels were available, offering 12 images with time intervals that are composed of arbitrary multiples of $2 \mathrm{~ns}$. The excitation pulses were then delayed in steps of 250 or 125 ps with respect to the photon 


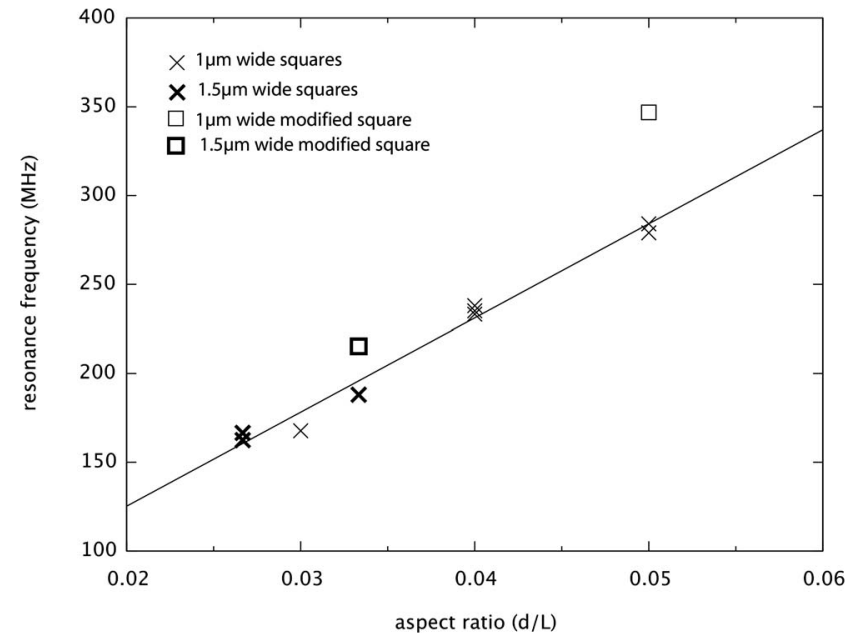

FIG. 3. Resonance frequency as a function of the aspect ratio $d / L$ (thickness/lateral size) of the investigated square-shaped structures. The frequencies of the unmodified structures (crosses) are fitted with a linear function $f_{0}=a \times d / L+b$. The resonance frequencies of the FIB modified squares (square markers) are increased due to the pinning of the domain walls.

flashes in order to achieve time steps smaller than $2 \mathrm{~ns}$. The temporal resolution in these measurements is limited by the width of the X-ray flashes and is about 100 ps.

One counting channel was used to record a reference image of the magnetic vortex configuration in its ground state before the magnetic field pulse. The remaining channels contain dynamic information and were divided by the reference image, leading, in this way, to a series of differential images. This procedure cancels the static magnetic contrast contributions and slow variations in the photon flux and retains only the dynamic magnetic signals.

Figure 2 shows a sequence of STXM images for a $1 \mu \mathrm{m} \times 1 \mu \mathrm{m} \times 50 \mathrm{~nm}$ FIB-modified square during one period of oscillation, as well as the corresponding differential images. The gyrotropic motion can be observed in the differential images by the sequence of black and/or white (or white and/or black) crosses, which indicate a vortex configuration displaced to the right (or left), and full bright (or dark) dots, corresponding to a vortex configuration displaced upward (or downward). A detailed interpretation of differential images can be found in Puzic et al. ${ }^{12}$ The absolute value of the integrated contrast will thus be maximal if the vortex core is fully displaced upward or downward and will vanish if the vortex core is only horizontally displaced. The integrated contrast over an area of the differential images exactly oscillates at the eigenfrequency of the gyration mode, as shown in Fig. 2(c). The resonance frequency can be determined precisely from the integrated differential contrast as a function of time.

\section{RESULTS}

The resonance frequencies of the square- and disk-shaped structures are presented in Figs. 3 and 4, respectively. The frequencies are shown as a function of the aspect ratio $d / L$

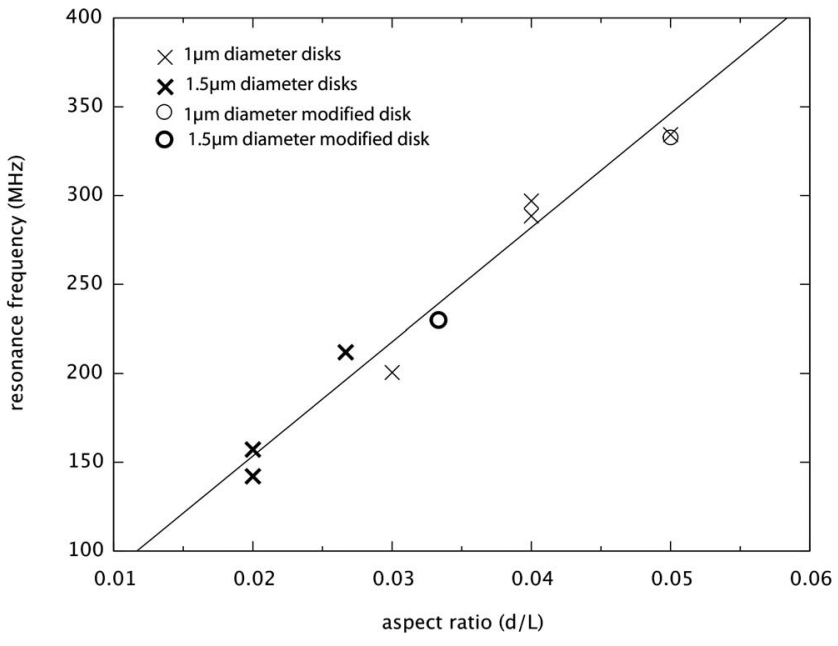

FIG. 4. Resonance frequency as a function of the aspect ratio $d / L$ (thickness/lateral size) of the investigated disk-shaped structures. The frequencies of the unmodified structures (crosses) are fitted with a linear function $f_{0}=a \times d / L+b$. The resonance frequency of the FIB modified disks (round markers) are not significantly changed.

(thickness/lateral size) of the structures, as this parameter has the largest influence on the resonance frequency $f_{0}$. For the unmodified structures, the data could be relatively fitted well with a linear function $f_{0}=a \times d / L+b$, although a small dependence on $d$ and $L$ can be separately observed as well. For the unmodified square structures, the following values were found: $a=5.30 \mathrm{GHz} \pm 5 \%$ and $b=19 \mathrm{MHz} \pm 48 \%$. For the unmodified disks, the linear fit gave $a=6.43 \mathrm{GHz} \pm 5 \%$ and $b=25 \mathrm{MHz} \pm 72 \%$, which approximately shows a linear scaling with the aspect ratio, which is consistent with the theoretical model of Guslienko et $\mathrm{al}^{4}{ }^{4}$

Two FIB modified squares are also plotted in Fig. 3: sample $\mathrm{S} 1$, which is a $1.5 \mu \mathrm{m} \times 1.5 \mu \mathrm{m} \times 50 \mathrm{~nm}$ square with four antidots at about $350 \mathrm{~nm}$ from the center (bold marker), and sample $\mathrm{S} 2$, which is a $1 \mu \mathrm{m} \times 1 \mu \mathrm{m} \times 50 \mathrm{~nm}$ square with four antidots at about $280 \mathrm{~nm}$ from the center (regular marker). In both cases, the resonance frequency is significantly higher than that for the unmodified structures. The increase is most noticeable for sample S2, which has the antidots closer to the center: the frequency is altered from $282 \pm 4$ to $346 \pm 4 \mathrm{MHz}$, an increase of about $23 \%$.

Figure 4 shows the resonance frequency of a 1.5- $\mu \mathrm{m}$-wide, 50 -nm-thick disk with four antidots at about $350 \mathrm{~nm}$ from the center (bold marker) and a 1- $\mu \mathrm{m}$-wide, 50 -nm-thick disk with four antidots at about $280 \mathrm{~nm}$ from the center (regular marker). These modifications are similar to the ones in the corresponding square-shaped structures. However, the disks showed no statistically significant alteration of the resonance frequency.

\section{DISCUSSION}

The STXM images of the square structures show that during the gyration, the domain walls do not leave the antidots and are thus pinned by these modifications. A comparison 


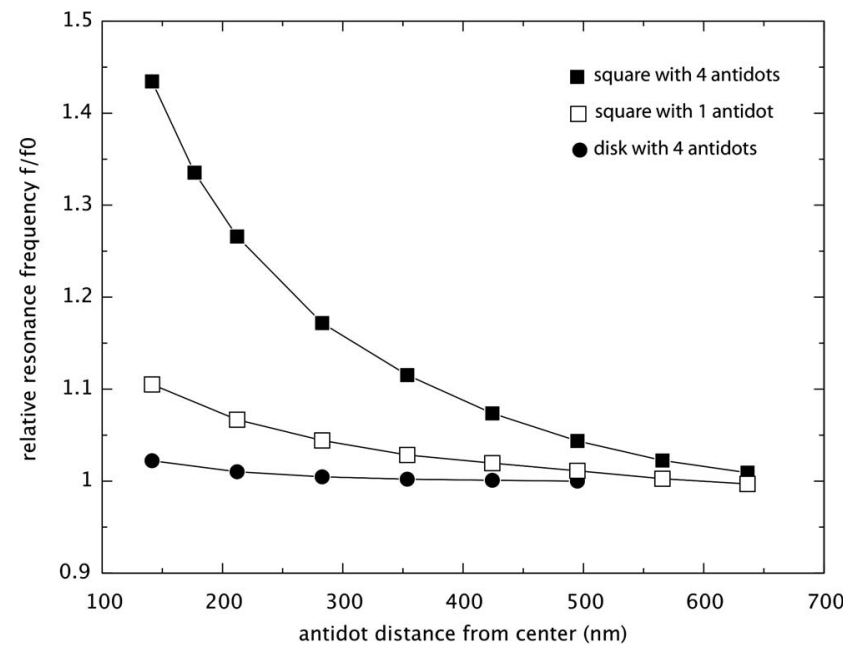

FIG. 5. Simulated vortex gyration frequencies for 1- $\mu$ m-wide, 50-nm-thick disk- and square-shaped modified structures, which are relative to the resonance frequency $f_{0}$ of the corresponding unmodified structures as a function of the distance of the modifications from the structure center. The square structures with four or one antidot(s) on the domain wall(s) have a resonance frequency that increases significantly when the modifications are moved closer to the center, while the resonance frequency of the disks is almost unaffected.

between Figs. 2(b) and 2(d) also suggests that the domain wall motion is mostly confined to a region inside the four antidots. This can be seen much clearer, however, in the simulated images in Fig. 5(a) where the domain walls only move in the area inside the antidots. This suggests that the domain wall pinning is the reason behind the higher resonance frequencies in square-shaped structures, which is confirmed by the control measurements on the disk-shaped structures. Since these structures do not have domain walls, the presence of the antidots does not affect the gyration frequency.

The experimental results are consistent with micromagnetic simulations ${ }^{14}$ carried out with the OOMMF (Ref. 13) package. 1- $\mu \mathrm{m}$-wide, 50-nm-thick squares and disks were simulated, and the antidots were introduced by setting the magnetization of four areas of $20 \times 20 \mathrm{~nm}^{2}$ to zero. The distance of the antidots from the structure center was also varied.

Figure 5 shows the simulated relative resonance frequencies $f / f_{0}$ as a function of the antidot distance to the structure center, where $f_{0}$ is the resonance frequency of the unmodified structure. The square structures undergo a significant increase in resonance frequency when the antidots are moved closer to the structure center. For a square with four antidots at $283 \mathrm{~nm}$ from the center, the increase is about $17 \%$, which is in the same order of magnitude as the corresponding experimental value of about $23 \%$.

For the disk-shaped structures, the resonance frequency is virtually unaltered by the presence of the antidots. Even if these are placed very close to the structure center, the increase is only a few percent. This is also in agreement with the experiments where no significant alteration of the resonance frequency is observed. As an illustration, simulation results for squares with only one antidot are presented as well, which also causes an increased resonance frequency. This indicates that a frequency increase is not limited to the case of four antidots, placed symmetrically on the diagonals of the square, but can generally be observed when one or more domain wall pinning sites are present.

The experimental results can also be intuitively understood. The energy of the domain walls is proportional to their length, which is reduced when the domain walls cross the antidots. During the vortex gyration movement, the domain walls do not leave the antidots and hence the movement of the domain walls is confined to a region inside the four antidots. This leads to an intuitive explanation of the increased eigenfrequency: the vortex inside the antidots behaves like a smaller Landau configuration, which has a higher eigenfrequency due to its higher aspect ratio.

The situation is, however, more complex since the material outside the antidots still influences the vortex dynamics. If this was not the case, the resonance frequency of sample S2 would be increased to about $750 \mathrm{MHz}$ (based on the aspect ratio) and not to $346 \mathrm{MHz}$. This can be understood if one considers that the restoring force, which drives the vortex gyration, is the magnetostatic field, which is induced by the displaced vortex (see Guslienko et al. $^{4}$ ). As the magnetization in the domains outside the antidots slightly adjusts to this magnetostatic field and partially closes the field lines, the effective magnetostatic field for the vortex is reduced (see Fig. 6). This implies a reduced restoring force acting on the vortex core, thus lowering the gyration frequency.

To summarize, the confinement of the domain wall motion to an area inside the antidots would lead to a rather large increase in the gyration frequency, but this effect is reduced because the domains outside the antidots are lowering the magnetostatic field acting on the displaced vortex core.

\section{CONCLUSIONS AND OUTLOOK}

In conclusion, we have used focused ion beam etching to create antidots in square- and disk-shaped Permalloy structures. In the case of the square-shaped structures, the domain walls were found to be pinned by the antidots, leading to a confinement of the domain wall motion to an area inside the antidots and an increase in the vortex gyration frequency. To demonstrate that the domain wall pinning is responsible for the higher frequency, disk-shaped structures with identical modifications were also measured. In these structures, no domain wall existed and, consequently, no alteration of the resonance frequency was observed. It was also intuitively explained how domain wall pinning leads to an increased gyration frequency: the area inside the antidots behaves as a smaller Landau configuration, with a higher gyration frequency due to its higher aspect ratio. This frequency increase is reduced, however, because the portion of the magnet outside the antidots reduces the magnetostatic field in which the vortex core gyrates. Micromagnetic simulations qualitatively agree with the experimental results and also show that the frequency increase rapidly grows as the antidots are moved 
(a)

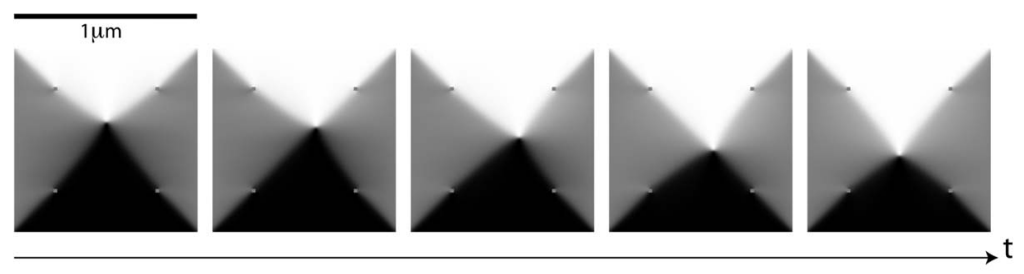

(b)

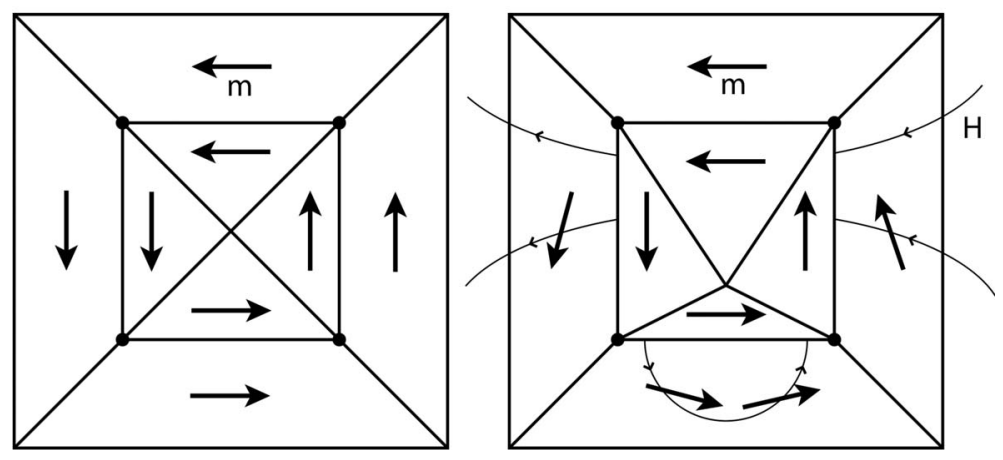

FIG. 6. (a) Simulation of half a period of the vortex gyration in a square-shaped structure with four antidots. The domain walls are pinned by the antidots leading to a confinement of the domain wall motion to an area inside the four antidots, which increases the gyration frequency. (b) Schematic representation of the simulated data for a configuration in the ground state (left) and during the gyration (right). The magnetic moments outside the four antidots were found to slightly tilt toward the direction of the magnetostatic field $H$, which was induced by the configuration inside the antidots. This reduces the magnetostatic field acting on the vortex core, which reduces the frequency increase.

closer to the structure center.

In square structures, naturally occurring magnetic defects of sufficient strength might also be able to trap domain walls. Although this has not yet been observed, in analogy to this work, one could expect that if such pinning sites are present, they could also increase the vortex gyration frequency.

\section{ACKNOWLEDGMENTS}

Financial support by The Institute for the promotion of
Innovation by Science and Technology in Flanders (IWTFlanders), by the German Research Foundation (DFG) through the priority program "Ultrafast Magnetization Processes," and by the Research Foundation Flanders (FWOFlanders) through the research Grant No. 60170.06 are gratefully acknowledged. The Advanced Light Source is supported by the Director, Office of Science, Office of Basic Energy Sciences, of the U.S. Department of Energy. The authors would also like to thank Aleksandar Puzic for his contributions to the experiments.
${ }^{1}$ T. Shinjo, T. Okuno, R. Hassdorf, K. Shigeto, and T. Ono, Science 289, 930 (2000).

${ }^{2}$ X. B. Zhu, Z. G. Liu, V. Metlushko, P. Grutter, and M. R. Freeman, Phys. Rev. B 71, 180408(R) (2005).

${ }^{3}$ K. Y. Guslienko, X. F. Han, D. J. Keavney, R. Divan, and S. D. Bader, Phys. Rev. Lett. 96, 067205 (2006).

${ }^{4}$ K. Y. Guslienko, B. A. Ivanov, V. Novosad, Y. Otani, H. Shima, and K. Fukamichi, J. Appl. Phys. 91, 8037 (2002).

${ }^{5}$ V. Novosad, F. Y. Fradin, P. E. Roy, K. S. Buchanan, K. Y. Guslienko, and S. D. Bader, Phys. Rev. B 72, 024455 (2005).

${ }^{6}$ C. E. Zaspel, B. A. Ivanov, J. P. Park, and P. A. Crowell, Phys. Rev. B 72, 024427 (2005).

${ }^{7}$ R. L. Compton and P. A. Crowell, Phys. Rev. Lett. 97, 137202 (2006).
${ }^{8}$ K. Kuepper et al., Appl. Phys. Lett. 90, 062506 (2007).

${ }^{9}$ A. Kilcoyne et al., J. Synchrotron Radiat. 10, 125 (2003).

${ }^{10}$ G. Schütz, W. Wagner, W. Wilhelm, P. Kienle, R. Zeller, R. Frahm, and G. Materlik, Phys. Rev. Lett. 58, 737 (1987).

${ }^{11}$ Y. Acremann, V. Chembrolu, J. P. Strachan, T. Tyliszczak, and J. Stohr, Rev. Sci. Instrum. 78, 014702 (2007).

${ }^{12}$ A. Puzic et al., J. Appl. Phys. 97, 10E704 (2005).

${ }^{13}$ M. Donahue and D. Porter, "OOMMF user's guide, version 1.0," National Institute of Standards and Technology Interagency Report No. NISTIR 6376, 1999 (unpublished).

${ }^{14} \mathrm{~A} 2 \mathrm{D}$ simulation of a $1 \mu \mathrm{m} \times 1 \mu \mathrm{m} \times 50 \mathrm{~nm}$ structure was used, with a grid size of $5 \mathrm{~nm}$ and material parameters $M_{s}$ $=736 \times 10^{3} \mathrm{~A} / \mathrm{m}, A=13 \times 10^{-12} \mathrm{~J} / \mathrm{m}, \alpha=0.02$, and $K_{1}=0$. 